\title{
Multi-Sensor Approach for the Monitoring of Halitosis Treatment via Lactobacillus brevis (CD2) - Containing Lozenges-A Randomized, Double-Blind Placebo-Controlled Clinical Trial
}

\section{Enrico Marchetti ${ }^{1, *}$, Simona Tecco ${ }^{2}$, Marco Santonico ${ }^{3}$, Chiara Vernile ${ }^{3}$, Daniele Ciciarelli ${ }^{1}$, Ester Tarantino ${ }^{1}$, Giuseppe Marzo ${ }^{1}$ and Giorgio Pennazza ${ }^{3}$}

1 Department MESVA, University of L'Aquila, Via Vetoio ed. Delta 6, 67100 L'Aquila, Italy; E-Mails: daky87@hotmail.it (D.C.),ester88x@hotmail.it (E.T.); giuseppe.marzo@cc.univaq.it (G.M.)

2 University Vita-Salute San Raffaele, I.R.R.C.S. San Raffaele Hospital, 20100 Milano, Italy; E-Mail: tecco.simona@hsr.it

3 Center for Integrated Research - CIR, Unit of Electronics for Sensor Systems, "Università Campus Bio-Medico di Roma", Via Alvaro del Portillo 21, 00128 Rome, Italy; E-Mails: m.santonico@unicampus.it (M.S.); c.vernile@unicampus.it (C.V.); g.pennazza@unicampus.it (G.P.)

* Author to whom correspondence should be addressed; E-Mail: enrico.marchetti@cc.univaq.it; Tel.: +39-86-243-4789.

Academic Editor: W. Rudolf Seitz

Received: 1 July 2015 / Accepted: 3 August 2015 / Published: 10 August 2015

\begin{abstract}
The aim of this randomized clinical trial was to evaluate whether a recently described multi-sensor approach called BIONOTE ${ }^{\circledR}$ is accurate enough to verify the efficacy of treatment of patients with halitosis. A treatment with Lactobacillus brevis (CD2)-containing lozenges, compared with placebo was tested. The BIONOTE ${ }^{\circledR}$ was compared with traditional techniques used to detect halitosis: OralChroma ${ }^{\mathrm{TM}}$ and two calibrated odor judges enrolled for the organoleptic assessments. Twenty patients (10 treated and 10 placebo), suffering from active phase halitosis were included in the study. Treatment consisted of Lactobacillus brevis (CD2) — containing lozenges or placebo, 4 tablets/day for 14 days. $t_{0}$ was before the beginning of the study; $t_{1}$ was day 7 and $t_{2}$ was day 14 . The effectiveness of treatment was assessed through: (1) Rosenberg score; (2) Winkel tongue coating index (WTCI) anterior and posterior; (2) OralChroma ${ }^{\mathrm{TM}}$; (3) the new developed multi-sensor
\end{abstract}


approach, called BIONOTE $^{\circledR}$ (test technique). Only the WTCI anterior revealed statistically significant changes between $t_{0}$ and $t_{2}$ data $(p=0.014)$ in the treated group. Except for the WTCI anterior, all diagnostic methods revealed the lack of effectiveness for halitosis of a 14-days treatment with Lactobacillus brevis (CD2)-containing lozenges. The BIONOTE $^{\circledR}$ multisensor system seems accurate in addition to OralChroma ${ }^{\mathrm{TM}}$ to assess the initial condition of halitosis and its mitigation during treatment.

Keywords: halitosis; bad breath; electronic nose; chemical sensors; sensor array; probiotics

\section{Introduction}

Non-selective gas sensor arrays are widely used, in a research context, for medical applications oriented to non-invasive prognosis, diagnosis and follow-up [1]. Despite promising results they cannot be used so far in the clinical practice [2]. Besides, therapy and treatment monitoring could be a feasible end-point for such technology [3]. It is worth remarking that in this preliminary phase the use of innovative techniques must be supported by consolidated procedures and instruments [4]. In the case of halitosis the OralChroma ${ }^{\mathrm{TM}}$ device and panellists are considered the standard [5]. This research has been designed with the objective of monitoring disease treatment and testing by using a recently developed multi-sensor approach, called BIONOTE ${ }^{\circledR}$.

In order to test the ability of this system to monitor halitosis treatment, Lactobacillus brevis (CD2)-containing lozenges were proposed to be tested, as a safe and effective treatment for periodontal diseases for various reasons, highlighted below [6].

The possible utility of Lactobacillus brevis (CD2) in the treatment of halitosis derives from arginine deiminase (AD), which has effects against halitosis by inhibiting the formation of volatile sulfur compounds (VSC) in the oral cavity. More specifically, Lactobacillus brevis (CD2) is able, through its arginine-deiminase activity, to removing the substrate (arginine) of nitric oxide (NO) synthase and polyamines (putrescine and spermidine). The inhibition of the formation of NO causes a significant inhibition of synthesis of prostaglandins, especially under inflammatory conditions, and blocks the increase in the production of proteolytic enzymes. This will limit the sources of peptides and amino acids for odorous bacteria, with the result of less formation of VSC and polyamines. The reduced amount of such cytotoxic agents facilitates ambient conditions more compatible with a good oral health status. Moreover, the limitation of the nutritional substances makes the growth of bacteria more difficult. In addition, the reduced formation of NO via the AD may counteract the inhibitory effect of lipopolysaccharide (LPS) of Gram-negative bacteria on the synthesis of salivary mucin, and the protective role of salivary mucin is known $[7,8]$.

Finally, the role of arginine to make bacteria resistant to acids, so that they can survive in an environment with variable acidity is well known. The depletion of the extracellular arginine by Lactobacillus brevis (CD2) could counteract the protective acidic neutralization of the bacteria, thus making them less resistant to changes in acidity [9].

The aim of this randomized clinical trial is to evaluate whether the recently described multi-sensor approach called BIONOTE ${ }^{\circledR}$ is accurate enough to verify the efficacy of treatment of patients with 
halitosis using Lactobacillus brevis (CD2)-containing lozenges, comparing the data with patients treated with placebo. The recently developed technique was tested through the comparison with traditional techniques: OralChroma ${ }^{\mathrm{TM}}$ and two calibrated odor judges enrolled for the organoleptic assessments.

\section{Experimental Section}

\subsection{Trial Design}

This is a monocenter, randomized, double-blind, placebo-controlled parallel-group study conducted in Italy. No changes to methods were done after trial commencement.

\subsection{Participants}

Possible patients for enrollment in the study were recruited by means of public posters and brochures in both the dental clinic of the University of L'Aquila, and pharmacies in the city of L'Aquila. Twenty patients were included in the study (10 treated with Lactobacillus brevis (CD2)-containing lozenges and 10 with placebo) suffering from active phase halitosis.

All the subjects who showed up for possible enrollment in the study were provided a free dental visit.

The eligibility criteria were:

(a) Adult age ( $>18$ years of age);

(b) Halitosis in active phase;

(c) Informed consent by the patient.

Exclusion criteria were:

(a) The need to take antibiotics for the presence of signs and/or symptoms of infection;

(b) Use of non-steroidal anti-inflammatory drugs during the 30 days prior to the beginning of the study;

(c) Use of steroid medications during the 30 days prior to the beginning of the study;

(e) Dental care in progress;

(f) Current gingivitis and periodontitis;

(g) Systemic diseases such as: chronic liver disease, chronic renal failure, gastro-esophageal reflux;

(h) Alcoholism and/or drug addiction.

The coexistence of other diseases was allowed, with the exception of those enumerated in the exclusion criteria. Informed consent was obtained from each patient included in the study.

\subsection{Study Setting}

The study took place at the Research Centre for the Diagnosis and Treatment of Halitosis, University of L'Aquila (L'Aquila, Italy) from January 2014 to June 2014. The Ethics Committee of the University of L'Aquila approved the protocol. 


\subsection{Interventions}

Patients received from the principal investigator (E.M.) three boxes, each containing 20 tablets (Lactobacillus brevis CD2 - containing lozenges or matching placebo).

The day after, patients started taking Lactobacillus brevis (CD2)-containing lozenges or placebo every day for 14 days, 4 tablets/day. With regard to additional therapies, if comorbidities were present, the use of other drugs was allowed, provided that - according to the investigator - this did not alter, in a significant manner, the patient's response to the treatment under study, and/or did not fall within the exclusion criteria listed in the Protocol. The principle investigator (E.M.) provided an explanation to each patient of the characteristics and the mode of administration of the drug.

The duration of the trial was established to be 14 days. At the end of this period, the experimenter (E.M.) provided the coordinator of the study (G.M.) with a report including data about the conduct and results of the trial. For the outcomes analysis, to was before the beginning of the study; $t_{1}$ was day 7 and $t_{2}$ was day 14 . At $t_{0}, t_{1}$ and $t_{2}$, the assessment of outcome measures was carried out.

The selection procedure from baseline screening to enrollment is described in Figure 1.

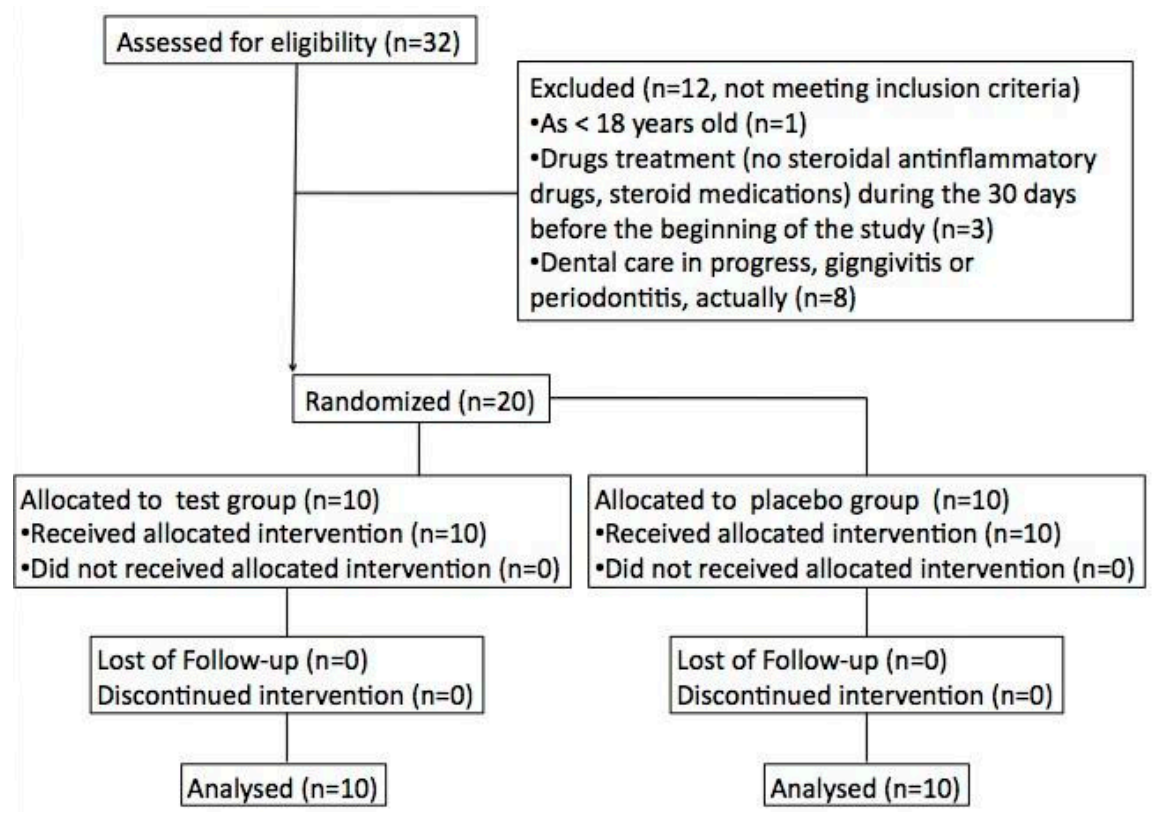

Figure 1. Flow diagram of the CONSORT 2010 study.

\subsection{Outcomes}

The primary endpoint with respect to efficacy in halitosis is the proportion of patients achieving an improvement in outcome measures from baseline $\left(\mathrm{t}_{0}\right)$ to day $7\left(\mathrm{t}_{1}\right)$ and day $14\left(\mathrm{t}_{2}\right)$.

Outcome measures are:

- $\quad$ the Breathprint (BP) obtained with the recently introduced technique, called BIONOTE ${ }^{\circledR}$ (at to, $\mathrm{t}_{1}$ and $\mathrm{t}_{2}$ ) (this is the "test technique", described above);

- the Rosenberg score [10], an organoleptic measurement assessed by two calibrated odor judges enrolled for the study, which is, at the actual state of the art, a gold standard, apart from the gas chromatography, in the diagnosis of halitosis; 
- $\quad$ the Winkel Tongue Coating Index, anterior and posterior (WTCI, anterior and posterior) [11] an index to evaluate the tongue coating, assessed by two calibrated operators enrolled for the study;

- $\quad$ the gas chromatography score (OralChroma ${ }^{\mathrm{TM}}$ ) [12].

No changes to trial outcomes after the trial commencement were decided. Additional analyses are done on the correlations among the different methods in targeting halitosis.

The recording of outcomes measurements began with the OralChroma ${ }^{\mathrm{TM}}$ because this tool takes 8 min for each analysis. Meanwhile the breath samples were collected for the BIONOTE ${ }^{\circledR}$ analysis. Finally, the organoleptic Rosenberg score and the WTCI score (anterior and posterior) were collected.

The day before the evaluation, the subject were forbidden to consume alcohol or smoke. Patients were asked not to eat, drink, smoke, nor perform oral hygiene for at least one hour before their appointment. Before the breath collection procedure, all patients were requested to rinse their mouth, without using toothpaste. During the assessment of the outcome measurements, the subjects avoided any rinsing between the collections. Globally, the assessment lasted about $10 \mathrm{~min}$. The outcome measures and how they were assessed are summarized in Table 1.

Table 1. Outcome measures and description of how they were assessed.

\begin{tabular}{|c|c|}
\hline Outcome Measures & Description of How Outcome Measures Were Assessed \\
\hline $\begin{array}{l}\text { Winkel Tongue Coating } \\
\text { Index (WTCI), anterior } \\
\text { and posterior }\end{array}$ & $\begin{array}{l}\text { The dorsum of the tongue was divided into six areas (three posterior, three anterior) and } \\
\text { tongue coating was assessed in each sextant as follows; } 0=\text { no coating, } 1=\text { light coating, } \\
2=\text { severe coating. The WTCI was obtained by adding all six scores, for a possible range } \\
\text { of } 0-6 \text { for WTCI anterior and } 0-6 \text { for WTCI posterior. }\end{array}$ \\
\hline $\begin{array}{l}\text { The Rosenberg score, } \\
\text { organoleptic measurement }\end{array}$ & $\begin{array}{l}\text { The organoleptic measurement depends on a trained examiner that has demonstrated } \\
\text { reliability in smelling halitosis. The operator, preferably blindfolded, sniffs the air } \\
\text { exhaled from the mouth at a distance of } 10 \mathrm{~cm} \text {. For recording a scale of } 5 \text { values is used. } \\
\text { The test is considered positive when the "hedonic" value assigned to breath exceeds the } \\
\text { number } 2 \text {. The scale includes the following values: } 0=\text { no odor; } 1=\text { doubtful presence of } \\
\text { halitosis; } 2=\text { slight odor but clearly notifiable; } 3=\text { moderate halitosis; } 4=\text { strong } \\
\text { halitosis; } 5=\text { very intense halitosis. }\end{array}$ \\
\hline $\begin{array}{l}\text { Gas chromatography } \\
\text { score, measured with } \\
\text { OralChroma }^{\mathrm{TM}}\end{array}$ & $\begin{array}{l}\text { Measure the molecular levels of the three major VSCs (hydrogen sulphide, methyl } \\
\text { mercaptan and dimethyl sulfide) in a sample of mouth air. The levels (measured in ppm) } \\
\text { are reported in a diagram from low to high level. A cognitive threshold is individuated } \\
\text { and levels are individuated as "more than" or "less than" the cognitive threshold. }\end{array}$ \\
\hline $\begin{array}{l}\text { BP (breath print) constructed } \\
\text { by BIONOTE }{ }^{\circledR}\end{array}$ & $\begin{array}{l}\text { Individual Breathprint (BP) of a patient is represented with a radar plot; equiangular } \\
\text { radii shape each radar plot, where each radius represents one of the } 28 \text { sensor responses. } \\
\text { The radius length gives magnitude of each sensor response (expressed in Hz, because } \\
\text { relative to a resonant frequency shoft of the quartz slice). The radar plot "profile" } \\
\text { consists of a line drawn connecting the data values for each radius. }\end{array}$ \\
\hline
\end{tabular}

The BIONOTE ${ }^{\circledR}$ technique has been described elsewhere [13]. Its characteristics can be thus summarized: the BIONOTE ${ }^{\circledR}$ (for BIOsensor-based multisensorial system for mimicking Nose, Tongue and Eyes) is a multisensory system recently introduced by two of the authors (G.P. and M.S.), able to analyse the volatile and liquid parts of a sample. In this clinical trial, only the gas sensor array is employed. The transducers used for the gas sensor array are seven quartz crystals with a resonance 
frequency of $20 \mathrm{MHz}$ in the thickness shear mode covered with a combination of anthocyanins extracted by three different plant tissues: red rose, red cabbage, and blue hortensia [14].

The final dataset of the gas analysis is composed of 28 responses due to the registration of the seven sensors' behaviour at four different temperatures of the sample (this procedure is dependent on the exhaled breath sampling on the adsorbent cartridge). The desorption of the cartridge into the sensors chamber is obtained by an interfacing device able to uniformly heat the tube from 50 to $200{ }^{\circ} \mathrm{C}$, and finally cleaning the cartridge holding the temperature at $300{ }^{\circ} \mathrm{C}$ for $5 \mathrm{~min}$. The final fingerprint of the exhaled breath is a sequence of four $n$-dimensional patterns, composed of the $n$ responses of an $\mathrm{n}$-dimensional gas sensor array at four temperatures (50-100-150-200 $\left.{ }^{\circ} \mathrm{C}\right)$. The individual Breathprint (BP, Figure 2) of a patient is represented with a radar plot; equiangular radii shape each radar plot, where each radius represents one of the 28 sensor responses. The radius length gives the magnitude of each sensor response. The radar plot "profile" consists of a drawn line connecting the data values for each radius.

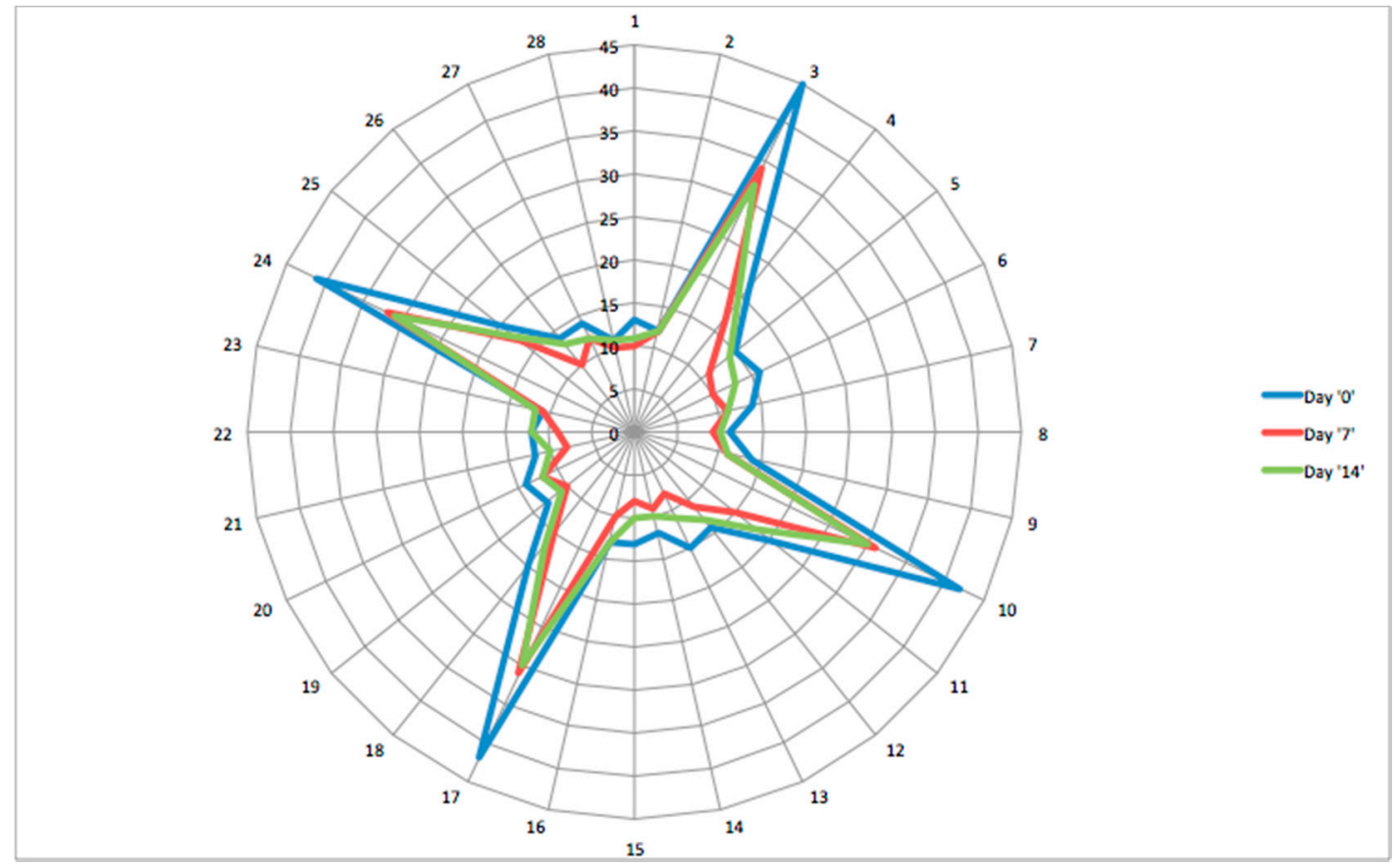

Figure 2. The Breathprint (BP) of one of the patients at day 0, day 7 and day 14 .

\subsection{Breath Collection}

Breath collection for the BIONOTE ${ }^{\circledR}$ analysis is performed through a procedure recently introduced by two of the authors (G.P. and M.S.) [15,16]. Each patient was asked to breathe for 3-min at tidal volume into a dedicated storage device for direct sampling of exhaled breath on an adsorbing cartridge [14]. The adsorbent cartridge used in this work was a Tenax GR by Supelco [17]. The samples (adsorbent cartridges) were then transported from L'Aquila to the Centre for Integrated Research - CIR, Unit of Electronics for Sensor Systems, University Campus Bio-Medico (Rome, Italy) where the BIONOTETM device is located.

No interim analyses were performed as the trial lasted 14 days. The stopping guidelines established that if a patient experienced side effects, a delay taking the study drug was planned. 


\subsection{Randomisation, Allocation and Blinding}

Participants were randomly assigned to treatment or placebo groups following simple randomization procedures (computerized random numbers). The allocation of treatment or placebo group was undertaken by a person not directly involved in the research project. The operator assessing outcomes and data collectors were blinded to the allocation of subjects.

Lactobacillus brevis (CD2)-containing lozanges and matched placebo were in lozenge form and had identical appearance. They were prepacked in boxes, each containing 20 tablets, with the same look and the same weight, so it was impossible to distinguish them a priori. The packages were consecutively numbered according to the randomisation schedule. Each participant was assigned an order number and received three boxes, each containing 20 tablets.

\subsection{Statistical Methods}

\subsubsection{Adverse Events}

The evaluation of the tolerability of Lactobacillus brevis (CD2) was based on the incidence and type of adverse events. Particular attention was given to any undesired event occurred during the 14 day testing period, reporting:

(a) Type of event;

(b) Characteristics of the undesired event;

(c) Date of onset;

(d) Duration (including whether it is expressed in minutes, hours or days);

(e) Maximum intensity reached;

(f) Mode of onset (immediate, gradual or asymptomatic);

(g) Possible therapy of the undesired event;

(i) Report of causality with the product;

(1) Outcome of the undesired event: if it disappeared without modification of the treatment or after stopping the treatment, or if it remained but the study could be continued.

\subsubsection{Effectiveness of the Treatment}

The assessment of the effectiveness of the treatment was carried out through with a multiparametric approach: (1) the parameters measured via the panellist assessment (Rosenberg score and WTCI, anterior and posterior); (2) the OralChroma ${ }^{\mathrm{TM}}$; (3) the BIONOTE ${ }^{\circledR}$ (test analysis).

The data analysis has been conducted following this flow-chart:

(1) Treatment efficacy assessment via each single technique:

a. By ANOVA test for panellist analysis: Rosenberg score, WTCI (anterior) and WTCI (posterior) ( $\mathrm{t}_{0}, \mathrm{t}_{1}$ and $\mathrm{t}_{2}$ assessments)

b. By partial least square - discriminate analysis (PLS-DA) for OralChroma ${ }^{\mathrm{TM}}$

c. By PLS-DA for BIONOTE ${ }^{\circledR}$

(2) Treatment efficacy assessment via a data fusion of the three techniques' outputs using PLS-DA 
(3) Correlation study between the three techniques

Partial least square discriminate analysis (PLS-DA) has been applied, with leave-one-out as cross-validation. In the case of small populations, in fact, the leave-one-out is a good cross-validation criterion using all the measurements of the dataset as training and test samples. PLS-DA was used as a regression method for calculating to which extent basal data could predict post-treatment data, and the differences between the study and the placebo groups. PLS-DA has been performed using the PLS-Toolbox SW (Eigenvector, Wenatchee, WA, USA) in the MATLAB environment (The Mathworks, Natick, MA, USA). A data pre-treatment was necessary for both data fusion and mapping, in order to overcome different dimensionalities of the data-sets: a linear normalization procedure has been used to this purpose.

\section{Results and Discussion}

\subsection{Results}

\subsubsection{Participant Flow}

No subject losses were registered. Eligible participants were recruited from January 2014 to June 2014. Participants attended clinic visits at the time of randomisation (baseline, to) and at 7-days intervals for 14 days. Baseline demographic data are reported in Table 2. The analysis involved all patients who were randomly assigned.

Table 2. Baseline demographic data.

\begin{tabular}{ccc}
\hline & $\begin{array}{c}\text { Lactobacillus brevis }(\mathbf{C D 2}) \text {-Containing } \\
\text { Lozenges }(\boldsymbol{n}=\mathbf{1 0})\end{array}$ & $\begin{array}{c}\text { Placebo } \\
(\boldsymbol{n}=\mathbf{1 0})\end{array}$ \\
\hline Age (mean years \pm SD) & $33 \pm 9$ & $36 \pm 7$ \\
Sex (males) & 12 & 11 \\
\hline
\end{tabular}

\subsubsection{Outcomes and Estimation}

(a) Treatment efficacy assessment with the different scores

The analysis only provided statistically significant differences over time for the test group treated with Lactobacillus brevis (CD2)-containing lozenges for the WTCI anterior test, and non-significant changes over time for the placebo group.

Specifically:

When the Rosenberg scores were analyzed, the ANOVA analysis revealed non-statistically significant changes over the time both for the placebo group $(p=0.074)$ and for the test group $(p=0.24)$. The same was true for the WTCI posterior test, for which the ANOVA analysis revealed non-statistically significant changes over time both for the placebo group $(p=0.074)$ and for the test group $(p=0.14)$. 
When the WCTI anterior data was analyzed, the ANOVA analysis revealed a statistically significant change over the time ( $\mathrm{t}_{0}, \mathrm{t}_{1}$ and $\left.\mathrm{t}_{2} ; p=0.037\right)$ for the test group; post-hoc analysis revealed statistically significant changes between $\mathrm{t}_{0}$ and $\mathrm{t}_{2}$ data $(p=0.014)$.

The PLS-DA model calculated on the OralChroma ${ }^{\mathrm{TM}}$ data showed different performances when used in different binary problems:

- Binary classification (0-7): correct classification of $50 \%$ of day 0 and of $80 \%$ of day 7 (see the confusion matrix reported in Figure 3a).

- Binary classification (0-14): correct classification of $50 \%$ of day 0 and of $90 \%$ of day 14 (see the confusion matrix reported in Figure 3b).

- $\quad$ Binary classification (7-14): non-significant results.

For the data obtained through the BIONOTE ${ }^{\circledR}$ the basal BPs of the 40 patients suffering from halitosis appeared heterogeneous in profile and intensity. In addition, the visual analysis of radar plots showed directional change in BPs after treatment in the test group. The PLS-DA model calculated on the BIONOTE ${ }^{\circledR}$ data showed different performance when used in different binary problems:

- Binary classification (0-7): correct classification of $90 \%$ of day 0 and of $50 \%$ of day 7 (see the confusion matrix reported in Figure 3c).

- Binary classification (0-14): correct classification of $100 \%$ of day 0 , and of $50 \%$ of day 14 (see confusion matrix reported in Figure 3d).

- $\quad$ Binary classification (7-14): not significant results.

\begin{tabular}{|l|l|l|l|}
\hline \multicolumn{2}{|c|}{} & \multicolumn{2}{|l|}{ predicted by OC } \\
\cline { 3 - 5 } \multicolumn{2}{|c|}{} & Day 0 & Day 7 \\
\hline \multirow{2}{*}{ actual } & Day 0 & 5 & 5 \\
\cline { 2 - 5 } & Day 7 & 2 & 8 \\
\hline
\end{tabular}

(a)

\begin{tabular}{|l|l|l|l|}
\hline \multicolumn{2}{|c|}{} & \multicolumn{2}{l|}{ ledicted byBionote } \\
\cline { 3 - 4 } \multicolumn{2}{|c|}{} & Day 0 & Day 7 \\
\hline \multirow{2}{*}{ actual } & Day 0 & 1 & 9 \\
\cline { 2 - 4 } & Day 7 & 5 & 5 \\
\hline
\end{tabular}

(c)

\begin{tabular}{|l|l|l|l|}
\hline \multicolumn{2}{|c|}{} & \multicolumn{2}{|l|}{ predicted by data-fusion } \\
\cline { 3 - 5 } \multicolumn{2}{|c|}{} & Day 0 & Day 7 \\
\hline \multirow{2}{*}{ actual } & Day 0 & 6 & 4 \\
\cline { 2 - 5 } & Day 7 & 2 & 8 \\
\hline
\end{tabular}

(e)

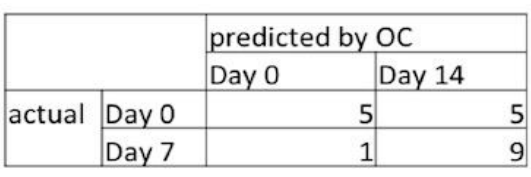

(b)

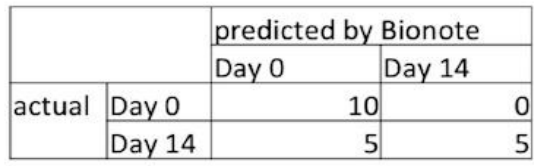

(d)

\begin{tabular}{|l|l|l|l|}
\hline \multicolumn{2}{|c|}{} & \multicolumn{2}{l|}{ predicted by data-fusion } \\
\cline { 3 - 5 } \multicolumn{2}{|c|}{} & Day 0 & Day 14 \\
\hline \multirow{2}{*}{ actual } & Day 0 & 5 & 5 \\
\cline { 2 - 5 } & Day 14 & 1 & 9 \\
\hline
\end{tabular}

(f)

Figure 3. Results of the experiment. Predicted cases at Day $0\left(t_{0}\right)$, Dat $7\left(t_{1}\right)$ and Day $14\left(t_{2}\right)$. OC: OralChroma ${ }^{\mathrm{TM}}$; (a,b) predicted by OralChroma ${ }^{\mathrm{TM}} ;(\mathbf{c}, \mathbf{d})$ predicted by $\mathrm{BIONOTE}^{\circledR}$; $(\mathbf{e}, \mathbf{f})$ predicted by data fusion. See text for details. 
(b) Treatment efficacy assessment via a data fusion of the three technique

When data fusion of the three techniques' outputs using the PLS-DA was analyzed, the PLS-DA model calculated on the data fusion of BIONOTE ${ }^{\circledR}$ and OralChroma ${ }^{\mathrm{TM}}$ outputs showed different trends in different problems. Specifically:

- Binary classification ( $\left.\mathrm{t}_{0}-\mathrm{t}_{1}\right)$ : correlated classification in $60 \%$ of cases at $\mathrm{t}_{0}$, and $80 \%$ at $\mathrm{t}_{1}$ (see the confusion matrix reported in Figure 3e);

- Binary classification ( $\mathrm{t}_{0}-\mathrm{t}_{2}$ ): correlated classification in $50 \%$ of cases at $\mathrm{t}_{0}$, and $90 \%$ at $\mathrm{t}_{2}$ (see the confusion matrix reported in Figure 3f);

- $\quad$ Binary classification ( $\left.\mathrm{t}_{1}-\mathrm{t}_{2}\right)$ : non-significant results.

\subsubsection{Correlation Study between the Three Techniques}

The BIONOTE ${ }^{\circledR}$ data allowed us to predict WCTI anterior, WCTI posterior and Rosenberg scores with a root mean square error in cross-validation (RMSeCV) of $0.52,0.73$ and 0.78 , respectively. The correlation between BIONOTE ${ }^{\circledR}$ and methylmercaptan $\left(\mathrm{CH}_{3} \mathrm{SH}\right)$ was $\mathrm{RMSeCV}=111$. The correlation between OralChroma ${ }^{\mathrm{TM}}$ and other techniques showed a rho $<0.25$. No adverse effects were registered.

Data analysis was performed in the MATLAB environment, by means of the PLS Toolbox. A partial least square discriminant analysis (PLS-DA) was performed, considering two classes in each model. In order to evaluate the identification performances of the method, a leave-one-out validation criterion has been adopted.

Table 3. Oral Chroma ${ }^{\mathrm{TM}}$.

\begin{tabular}{cccccccc}
\hline & RMSEC & RMSEC & RMSECV & RMSECV & \multirow{2}{*}{ LV1 } & LV2 \\
& LV1 & LV2 & LV1 & LV2 & & PRESS & PRESS \\
\hline Binary classification (0-7) & 0.45 & 0.43 & 0.49 & 0.48 & 2 & 4.88 & 4.74 \\
Binary classification (0-14) & 0.46 & 0.45 & 0.542 & 0.541 & 2 & 5.89 & 5.87 \\
Binary classification (7-14) & 0.44 & 0.43 & 0.47 & 0.49 & 2 & 6.7 & 7.2 \\
\hline
\end{tabular}

Table 4. BIONOTE ${ }^{\circledR}$.

\begin{tabular}{cccccccc}
\hline & RMSEC & RMSEC & RMSECV & RMSECV & \multirow{2}{*}{ LV1 } & LV2 \\
& LV1 & LV2 & LV1 & LV2 & & PRESS & PRESS \\
\hline Binary classification (0-7) & 0.43 & 0.41 & 0.45 & 0.44 & 2 & 4.75 & 4.66 \\
Binary classification (0-14) & 0.44 & 0.43 & 0.47 & 0.46 & 2 & 4.89 & 4.75 \\
Binary classification (7-14) & 0.56 & 0.54 & 0.51 & 0.54 & 2 & 6.4 & 7.1 \\
\hline
\end{tabular}

Table 5. Data fusion.

\begin{tabular}{cccccccc}
\hline & RMSEC & RMSEC & RMSEC & RMSECV & \multirow{2}{*}{ LV1 } & LV2 \\
& LV1 & LV2 & V LV1 & LV2 & & PRESS & PRESS \\
\hline Binary classification (0-7) & 0.61 & 0.54 & 0.50 & 0.51 & 2 & 8.77 & 6.43 \\
Binary classification (0-14) & 0.60 & 0.58 & 0.66 & 0.59 & 2 & 8.79 & 7.11 \\
Binary classification (7-14) & 0.53 & 0.50 & 0.55 & 0.53 & 2 & 8.32 & 8.58 \\
\hline
\end{tabular}


Model parameters are reported above. Each parameter is extracted from the model. The number of latent variables is selected considering a decrease of RMSEC, RMSECV and the PRESS. In particular the PRESS of binary classifications (7-14) increase for the second latent variable in all cases which a typical condition of overfitting (Tables 3-5).

\subsection{Discussion}

The short follow-up duration is a limitation of our study. As the participants were recruited among subjects who voluntarily answered the call that took place through brochures - so presumably the subjects that responded had a psychological interest in addressing the problem of halitosis - the present findings seem to be applicable for this class of subjects.

The primary outcome of this study was the evaluation of the accuracy of a recently developed system - the BIONOTE ${ }^{\circledR}$ — in the assessment of treatment outcomes, in comparison with traditional systems, such as OralChroma ${ }^{\mathrm{TM}}$, and two calibrated odor judges enrolled for the organoleptic assessments (a).

In addition, the efficacy of Lactobacillus brevis (CD2)-containing lozenges in the treatment of halitosis, comparing data with a placebo, was evaluated (b).

(a) The evaluation of the accuracy of a recently developed system - the BIONOTE ${ }^{\circledR}$ - in the assessment of treatment outcomes

When the accuracy of the methods was assessed via a data fusion of the techniques, the results suggested a proper utilization of the BIONOTE $^{\circledR}$ in addition to OralChroma ${ }^{\mathrm{TM}}$ consisting of their combination to assess the initial condition of halitosis (diagnosis at $t_{0}$ ) by BIONOTE $^{\circledR}$ and its mitigation during treatment by OralChroma ${ }^{\mathrm{TM}}$.

The good results obtained by data fusion (Figure 3e,f) suggested verifying whether these multidimensional systems could predict the traditional panellist indexes. BIONOTE ${ }^{\circledR}$ data allowed us to predict WTCI anterior, WCTI posterior and Rosenberg scores, with a root mean square error in cross-validation (RMSeCV) of $0.52,0.73$ and 0.78 , respectively, while the correlation between OralChroma $^{\mathrm{TM}}$ and panellists' indexes showed a rho $<0.25$.

(b) Efficacy of Lactobacillus brevis (CD2) — containing lozenges in the treatment of halitosis

The WTCI anterior showed statistically significant changes between $\mathrm{t}_{0}$ and $\mathrm{t}_{2}(p=0.014)$ in the treated group. These results suggest a significant effect of Lactobacillus brevis (CD2)-containing lozenges in reducing tongue coating and the total number of bacteria (mostly Fusobacterium nucleatum) on the dorsal surface of the tongue responsible for VSC.

A possible explanation could be that Lactobacillus brevis (CD2) can reverse (or attenuate) the inhibitory effect of LPS on the formation of mucin, so restoring the "washing" effects of mucin in this area. Another explanation could be that with the increase of the extracellular $\mathrm{pH}$ due to the introduction of AD during therapy, Fusobacterium nucleatum increases the use of glucose [18], instead of aminoacids [19], with a consequent reduction of the formation of VSC from Fusobacterium nucleatum on the dorsal suface of tongue. In addition, the odorous bacteria on the dorsal surface of the tongue produce methyl mercaptan from methionine, using arginine [20]. The reduction, by CD2, of the 
substrate (arginine) can consequently reduce intracellular polyamines - such as putrescine, spermidine and spermine $[21,22]$.

Apart from WTCI, the other methods did not evidence statistically significant changes over the duration of the treatment. Consequently, the tested treatment only showed a significant efficacy in the treatment of the dorsal surface of the tongue. The study failed to evidence a statistically significant effect of the tested treatment on halitosis after 14 days of treatment. Long-term efficacy of this treatment cannot be excluded, also considering the small sample of this study, and the fact the initial halitosis values were not very serious.

\section{Conclusions}

The aim of this randomized clinical trial was to validate a new multi-sensor approach called BIONOTE $^{\circledR}$ in the monitoring of treatment of halitosis, in comparison with traditional techniques, and to evaluate the clinical efficacy of Lactobacillus brevis (CD2)-containing lozenges in the treatment of halitosis. The multisensor system BIONOTE ${ }^{\circledR}$ can be used in addition to OralChroma ${ }^{\mathrm{TM}}$ to assess the initial condition of halitosis (diagnosis at $t_{0}$ ) by BIONOTE $^{\circledR}$ and its mitigation during treatment by OralChroma ${ }^{\mathrm{TM}}$.

The study failed to evidence a statistically significant efficacy of Lactobacillus brevis (CD2)-containing lozenges in the treatment of halitosis after 14 days, although a statistically significant reduction of tongue coating on the dorsal surface of tongue (WTCI anterior) was observed in the test groups, compared to the placebo group. Long-term efficacy of the treatment cannot be excluded, as this trial only lasted 14 days.

\section{Acknowledgments}

The authors wish to thank Florence Pryen and the company CD Investment for providing the supply of products for tests, and Vito Trinchieri for his help in the protocol definition.

\section{Author Contributions}

Each author contributed extensively to the preparation of this manuscript. E.M. conceived and designed the experiments. He also has made a substantial contribution to the writing of the paper; S.T. contributed to the interpretation of data and the writing of the paper; M.S. contributed to the manufacture of the instruments and has supervisioned the measurement processes; C.V. contributed to the samples collection and measurement and to the data analysis process; D.C. and E.T. performed the breath sample collection; G.M. revised critically the manuscript; G.P. contributed by coordinating instruments fabrication and set-up. He also has contributed to data analysis and interpretation. All authors participated in the discussion about the proposal and contributed to the analysis of the results.

\section{Conflicts of Interest}

The authors declare no conflict of interest. 


\section{References}

1. Scarlata, S.; Pennazza, G.; Santonico, M.; Pedone, C.; Antonelli Incalzi, R. Exhaled breath analysis by electronic nose in respiratory disease. Expert Rev. Mol. Diagn. 2015, 15, 933-956.

2. Beauchamp, J.D.; Pleil, J.D. Simply breath-taking? Developing a strategy for consistent breath sampling. J. Breath Res. 2013, 7, doi:10.1088/1752-7155/7/4/042001.

3. Seesaard, T.; Lorwongtragool, P.; Kerdcharoen, T. Development of fabric-based chemical gas sensors for use as wearable electronic noses. Sensors 2015, 15, 1885-1902.

4. Pennazza, G.; Santonico, M.; Finazzi Agrò, A. Narrowing the gap between breathprinting and disease diagnosis, a sensor perspective. Sens. Actuators B Chem. 2013, 179, 270-275.

5. Salako, N.O. Philip, L. Comparison of the use of the Halimeter and the Oral Chroma in the assessment of the ability of common cultivable oral anaerobic bacteria to produce malodorous volatile sulfur compounds from cysteine and methionine. Med. Princ. Pract. 2011, 20, 75-79.

6. Riccia, D.N.; Bizzini, F.; Perilli, M.G.; Polimeni, A.; Trinchieri, V.; Amicosante, G.; Cifone, M.G. Anti-inflammatory effects of Lactobacillus brevis (CD2) on periodontal disease. Oral Dis. 2007, 4, 376-385.

7. Slomiany, B.L.; Murty, V.L.N.; Piotrowski, J. Salivary Mucins in Oral Mucosal Defense. Gen. Pharmacol. 1996, 27, 761-771.

8. Slomiany, B.L.; Slomiany, A. Porphyromonas gingivalis lipopolysaccharide interferes with salivary mucin synthesis through inducible nitric oxide synthase activation by ERK and p38 kinase. Biochem. Biophys. Res. Commun. 2002, 297, 1149-1153.

9. Casiano-Colon, A.; Marquis, R.E. Role of the arginine deiminase system in protecting oral bacteria and an enzymatic basis for acid tolerance. Appl. Environ. Microbiol. 1988, 54, 1318-1324.

10. Rosenberg, M.; McCulloch, C.A. Measurement of oral malodor: Current methods and future prospects. J. Periodontol. 1992, 63, 776-782.

11. Winkel, E.G.; Roldan, S.; van Winkelhoff, A.J.; Herrera, D.; Sanz, M. Clinical effects of a new mouthrinse containing chlorhexidine, cetylpyridinium chloride and zinc-lactate on oral halitosis. J. Clin. Periodontol. 2003, 30, 300-306.

12. Tangerman, A.; Winkel, E.G. The portable gas chromatograph OralChroma ${ }^{\mathrm{TM}}$ : A method of choice to detect oral and extra-oral halitosis. J. Breath Res. 2008, 2, doi:10.1088/1752-7155/2/1/017010.

13. Santonico, M.; Pennazza, G.; Grasso, S.; D’Amico, A.; Bizzarri, M. Design and test of a biosensor-based multisensorial system: A proof of concept study. Sensors 2013, 13, 16625-16640.

14. Antonelli Incalzi, R.; Pennazza, G.; Scarlata, S.; Santonico, M.; Vernile, C.; Cortese, L.; Frezzotti, E.; Pedone, C.; D'Amico, A. Comorbidity modulates non invasive ventilation-induced changes in breath print of obstructive sleep apnea syndrome patients. Sleep Breath. 2014, 19, 623-630.

15. Pennazza, G.; Santonico, M.; Antonelli Incalzi, R.; Scarlata, S.; Chiurco, D.; Vernilea, C.; D'Amico, A. Measure chain for exhaled breath collection and analysis: A novel approach suitable for frail respiratory patients. Sens. Actuators B Chem. 2014, 204, 578-587.

16. Pennazza, G.; Santonico, M.; D’Amico, A.; Antonelli Incalzi, R.; Petriaggi, M. Pneumopipe-Auxiliary Device for Collection and Sampling of Exhaled Air. Domanda EU 12425057.2, 25 September 2013. 
17. Tenax ${ }^{\circledR}$ GR Adsorbent Resin for Trapping Volatiles. Scientific Instrument Services, Inc. Available online: http://www.sisweb.com/index/referenc/ tenaxgrm.htm (accessed on 20 May 2011).

18. Kapatral, V.; Anderson, I.; Ivanova, N.; Reznik, G.; Los, T.; Lykidis, A.; Bhattacharyya, A.; Bartman, A.; Gardner, W.; Grechkin, G.; et al. Genome Sequence and Analysis of Oral Bacterium Fusobacterium nucleatum Strain ATCC 25586. J. Bacteriol. 2002, 184, 2005-2018.

19. Zilm, P.S.; Gully, N.J.; Rogers, A.H. Growth $\mathrm{pH}$ and transient increases in amino acid availability influence polyglucose synthesis by Fusobacterium nucleatum grown in continuous culture. FEMS Microbiol. Lett. 2002, 215, 203-208.

20. Wallace, H.M.; Fraser, A.V.; Hughes, A. A perspective of polyamine metabolism. Biochem. J. 2003, 376, 1-14.

21. Lieber, C.S.; Packer, L. S-Adenosylmethionine: Molecular, biological, and clinical aspects-An introduction. Am. J. Clin. Nutr. 2002, 76, 1148S-1150S.

22. Yerlikaya, A. Polyamines and S-Adenosylmethionine Decarboxylase. Turk. J. Biochem. 2004, 29, 208-214.

(C) 2015 by the authors; licensee MDPI, Basel, Switzerland. This article is an open access article distributed under the terms and conditions of the Creative Commons Attribution license (http://creativecommons.org/licenses/by/4.0/). 